\title{
The Influence of Mindfulness on the Decreasing Anxiety in Nursing Students to Support Academic Learning: A Literature Review
}

\author{
Reza Indra Wiguna ${ }^{1}$, Meidiana Dwidiyanti ${ }^{2}$, Sri Padma Sari ${ }^{3}$ \\ ${ }^{I}$ Student of Master Program in Nursing, Faculty of Medicine, Diponegoro University, Indonesia \\ 2,3 Departement of Nursing, Faculty of Medicine, Diponegoro University, Indonesia \\ Corresponding Author: Meidiana Dwidiyanti (mdwidiyanti@gmail.com)
}

\begin{abstract}
Background: Anxiety and stress in nursing students has been shown to result in a lack of concentration, reduced learning productivity, and unfavorable physiological effects. This can cause a bad risk to the academic process of nursing students. A positive coping mechanism to reduce anxiety can be performed through various mindfulness training to support the academic success of nursing students.

Purpose: This literature review study aimed to describe the influence of mindfulness on decreasing anxiety in nursing students to support academic learning, from various literature studies.

Method: This study is a literature review. Data were collected through the online databases of EBSCO, PubMed, and ScienceDirect. The articles used in this study were determined published in 2010 to 2017. The key words used in searching for the articles were mindfulness, mindfulness in nursing students, nursing student anxiety, and nursing student achievement.

Results: The results of this literature indicate that mindfulness is a holistic intervention that significantly improves mental health in nursing students. It can support students' learning productivity to improve their academic achievement. The focus of mindfulness training program is to develop a better way to observe and concentrate, which in turn will facilitate a reduction in suffering and increased inner peace. Mindfulness will create relaxed attitudes and facilitates an open acceptance of experiences, thoughts, and learning.

Conclusion: Mindfulness therapy with various types of exercises would be a good alternative for nursing students to reduce anxiety. Future studies should explore the long-term effects of mindfulness on the students' learning productivity and academic achievement.
\end{abstract}

Keywords: Anxiety; academic learning; mindfulness; nursing students

\section{BACKGROUND}

A transition to college life requires adults to adapt to new environments, social situations, academic workload, and some other aspects. As students attempt to acclimate to new environments and situations, stress and anxiety often occur. Anxiety and depression are often experienced simultaneously. Anxiety affects about $67 \%$ of college 
students and becomes a major predictor of depression for nursing students (Dawood, 2016; Song \& Lindquist, 2015).

Nursing is a demanding profession with prolonged levels of stress and anxiety that account for high attrition rates observed among newly graduated nurses; attrition that negatively impacts on patient care and increases costs. Increased stress begins in school. Students in undergraduate nursing programs experience high levels of stress and anxiety in competitive programs of study (Turner \& Mccarthy, 2016). The academic environment today demands the attention of the students. As a result, some problems are encountered and most likely lead to behaviors that can negatively impact academic achievement (S. L. Shapiro, Schwartz, \& Bonner, 2013).

When comparing nursing students to other students in health education, the nursing students have higher rates of stress and anxiety, which manifests as higher rates of psychological and physiological symptoms. The cause of such high levels of stress has been directly related to the fact that nursing students must perform both academically and clinically to successfully be allowed to practice nursing (Alzayyat \& Al-Gamal, 2014; Manocchi, 2017). Sources of anxiety and stress among nursing students can be varied. Academic sources include examinations, fear of failure and workload. Clinical sources include clinical placements, fear of making mistakes and interactions with other staff members. Personal and social sources include financial concerns and the absence of leisure time (Alzayyat \& Al-Gamal, 2014; Pryjmachuk \& Richards, 2007).

Anxiety is a symptom of concern that is unclear and is associated with feelings of uncertainty and helplessness (Floyd, Clarke, Ed, Clarke, \& Ed, 2015). Mild anxiety can have a positive influence on the student; mild levels of anxiety can increase efficiency and intellectual functioning. However, high levels of anxiety are detrimental to academic outcomes. In fact, while $56.9 \%$ of students experienced 'overwhelming anxiety' in the previous twelve months, $21.9 \%$ of college students reported that anxiety negatively impacted their academics (ACHA-NCHA, 2013). Students with both decreased aptitude for academics and high levels of anxiety are more likely to have poor study skills and academic outcomes; placing them at an increased risk of failure (Bamber \& Schneider, 2016).

If students do not learn to handle these emotional demands appropriately, then when they encounter challenges as newly graduated nurses, they may feel overwhelmed during the first year of their new profession (Dubert, Schumacher, Jr, Gutierrez, \& Barnes, 2016). These high levels of anxiety and emotion are identified to impede memory as well as concentration and problem-solving abilities, which may also impede academic performance and learning (Beddoe \& Murphy, 2004). Thus, nursing students need to know how to manage their stress and emotions. A program of teaching selfmanagement of anxiety such as mindfulness may provide benefits to the students in their academic program and, if practiced, may enhance their future professional nursing practice (Song \& Lindquist, 2015).

Among the many methods of meditation, mindfulness is a nonjudgmental awareness of moment-to-moment experiences. Mindfulness is one such means that has been shown to 
reduce stress and anxiety in numerous research studies on both healthy and chronically ill individuals (Manocchi, 2017). Mindfulness is defined as "paying attention in a particular way: on purpose, in the present moment, and non-judgmentally" (Kabat-zinn, 2003). That is to say, in mindfulness practice, individuals are encouraged to pay attention to what is happening in the moment, in a non-judgmental way, without relying on previously schemas. This approach may eventually help change the way students think (Li, Yuan, \& Zhang, 2015).

Mindfulness meditation is a form of mental training that includes a students of nursing that incorporate a meditative component; in this approach, individuals are trained to consciously calm their minds to still or empty the mind and ultimately achieve inner peace or harmony (Chen, Yang, Wang, \& Zhang, 2013; Travis \& Pearson, 2000). Mindfulness meditation offers an accessible, low-cost, therapeutic approach that avoids the stigma of psychiatric treatments (Chen et al., 2013). Recently, researchers studied the effects of mindfulness meditation on the general public in Korea (Sik, Young, \& Ryu, 2009). Few studies, however, have evaluated its effects on nursing students, and can also affect the students' ability to provide comprehensive nursing care for patients (Li et al., 2015).

\section{OBJECTIVE}

The purpose of this review is to see how far the influence of mindfulness therapy on the anxiety level of nursing students to support the learning activities in academic as well as in clinical practice.

\section{METHODS}

The databases that were utilized in this literature search include EBSCOhost, PubMed, and ScienceDirect. To expand the topic of the literature search, the articles is determined by the year 2010 to 2017. The criteria and key words used for the main topic search included mindfulness, mindfulness in nursing students, nursing student anxiety, and nursing student achievement. From the conceptual framework of the literature that has been made, Mindfulness Based Stress Reduction (MBSR) and Mindfulness Meditation (MM), were added to the search in order to research current articles that pertained to both mindfulness and student nurse anxiety.

Table 1. Summary studies of the mindfulness reviewed

\begin{tabular}{ll}
\hline Study characteristic & No. of studies \\
\hline Date of publication & \\
Before 2015 & 3 articles \\
After 2015 & 5 articles \\
Research design & 2 articles \\
RCT & 6 articles \\
Non RCT & \\
Intervention & \\
MBSR & 3 articles \\
\hline
\end{tabular}


HOLISTIK NURSING AND HEALTH SIENCE 1, (1), 2018 23-33

\begin{tabular}{cl}
\hline Study characteristic & No. of studies \\
\hline MM & 2 articles \\
FMI & 1 articles \\
\hline
\end{tabular}

\section{RESULTS}

A literature review of the anxiety of nursing students is still limited. However, there are some interesting articles for review to obtain scientific evidence. Manocchi (2017) discussed in her literature that the incidence of anxiety in nursing students is very vulnerable to occur caused by many factors that influence it. Anxiety and depression are often experienced simultaneously.

Shapiro et al., (2013) identified that stress among nursing students is particularly high because of the clinical and classroom demands, especially during the first year of academic study. Clinical performance is also a large stressor for first year nursing student because the first year nursing student enters into the clinical placement for the very first time (A. L. Shapiro, 2014; S. L. Shapiro et al., 2013). Sun et al., 2016 did link heightened levels of anxiety in nursing students to poor health, attention difficulties, decreased the ability to make decisions, and poor cognitive function.

Depression affects about $67 \%$ of college nursing students with anxiety, and anxiety was a major predictor of depression for nursing students (Song \& Lindquist, 2015). Another study conducted on nurse students at Riyadh showed that only $14.4 \%$ of students demonstrated severe test anxiety; about $50.9 \%$ experienced moderate test anxiety and more than one-quarter of the participant's experienced mild test anxiety (Dawood, 2016).

In a study on anxiety specific to health-related disciplines, i.e., medicine, nursing, pharmacy, and social work $(n=552)$, Spadaro (2016) found that nursing students experienced higher levels of anxiety with more physical and psychological symptoms compared to the other majors (Spadaro \& Hunker, 2016).

Melo et al., (2010) investigated that nursing students' have shown specific clinical stressors including conflict with instructors, stressful work environments, fear of making mistakes, patient death, a lack of professional proficiency and difficulty in developing relationships with other professionals.

Anxiety is a common phenomenon that is a universal cause of poor academic performance among students worldwide. It is a common irrefutable fact in human life that affects the achievement of a person in various situations, the level of average anxiety is useful to help people work hard (Dawood, 2016). Nursing students experience many emotional demands such as fear of making a mistake with patients, financial and time constraints, and adapt their personal life with school requirements (Cook, 2005; Melo et al., 2010). High levels of anxiety and clinical levels of anxiety among nursing students is of concern because of its negative association with learning and clinical performance, burnout, attrition rates in schools of nursing (Melo et al., 2010). 
The purpose of this study is to see from a wide range of literature from various types of mindfulness to overcome the above problems, and determine the mechanisms emotion regulation strategies among nursing students. Thus, nursing students need to know how to manage their anxiety problem.

There are many strategies to solve the problem of anxiety and regulate emotions in order to calm ourselves. Medications have been shown to reduce the stress response; however, it was identified that such medications as benzodiazepines, which are frequently prescribed for anxiety and stress, are overused and highly addictive (Platt, Whitburn, Platt-Koch, \& Koch, 2016). Nursing students are taught that alternative forms of healing revolve around the mind- body connection, particularly in relation to coping. Finding holistic ways in which to assist with stress and anxiety is necessary (Manocchi, 2017).

Mindfulness is one such means that has been shown to reduce stress and anxiety in numerous research studies on both healthy and chronically ill individuals (Manocchi, 2017). In a Randomised Controlled Trial study conducted by Song \& Lindquist (2015) with Mindfulness Based Stress Reduction (MBSR) intervention in 44 Korean nursing students, it was found that the program of MBSR was effective when used in nursing students. The study reported significantly greater decreases in depression, anxiety and stress, and a greater increase in mindfulness. MBSR shows promising for use with nursing students to address their experience of mild depression, anxiety, and stress, and to increase mindfulness in academic and clinical work (Song \& Lindquist, 2015).

In other research results with 45 nursing students in the USA, it was found that mindfulness training improved mindfulness and some aspects of ethical decision making in the groups studied as part of this project (Sanko, Dnp, \& Ms, 2016). The effect of mindfulness was also studied in Chen et al., (2013), and the results found that brief mindfulness meditation was beneficial for Chinese nursing students in reducing anxiety symptoms and lowering systolic blood pressure. The findings of this study are promising and further demonstrate the merits of a mindfulness practice.

Student nurses must be prepared to face any problem to support their learning process. A program of teaching self-management of anxiety such as mindfulness may provide benefits to the nursing students in their academic program and, if practiced, it may enhance their future professional nursing practice (Song \& Lindquist, 2015).

\section{Mindfulness}

Mindfulness may be defined as 'paying attention on purpose, in the present moment, and non-judgmentally to the unfolding of experience moment by moment' (Kabat-zinn, 2003). It demands the regulation of attention and an orientation to present-moment curiosity, openness, and acceptance (Bishop \& Bishop, 2004). Mindfulness was developed by Kabat-Zinn at the University of Massachusetts Medical Centre in 1979 and has become the most commonly used mindfulness-based interventions; it also has standardized techniques, (Li et al., 2015). 
There are several types of mindfulness therapy such as Mindfulness Based Stress Reduction (MBSR) and Mindfulness Meditation. MBSR is one type of therapy of mindfulness commonly used as an intervention of research. MBSR program is conducted as an 8 to the 10-week course, meeting 2-2.5 hours weekly coupled with home practice most days. An all-day intensive mindfulness session for $7-8 \mathrm{~h}$ in one day is held around the sixth week (Song \& Lindquist, 2015).

MBSR programs are based on teaching participants to react nonjudgmentally to stressful events by focusing on automatic and dynamic stimuli (breath; body; eating; walking), and participants cultivate these skills (Zeidan, Johnson, Diamond, David, \& Goolkasian, 2010). MBSR programs have been studied, and scientific evidence has been generated demonstrating that they can have a profound benefit via the mind-body connection; the practice of mindfulness results in an increase of awareness (Kabat-zinn, 2003).

Whereas mindfulness meditation (MM) has been used traditionally for the systematic development of mindfulness; it essentially involves focusing on the mind at present, paying attention on purpose, in a moment-to-moment, nonjudgmental and nonreactive way (Chen et al., 2013). As a clinical intervention strategy based on Buddhist philosophy, mindfulness meditation has been applied in diverse groups to enhance wellbeing and enable individuals to cope with stress (Bamber \& Schneider, 2016).

Mindfulness Meditation is a mental practice based on focusing on the sensations of the breath/body while maintaining a relaxed state of mind. During formal meditation practice, distractions will arise, and the meditator is taught to acknowledge discursive thoughts, and non-judgmentally return his/her attention to their breathing (Wallace, 2006). Mindfulness meditation training was provided on seven consecutive days after the pre-treatment assessment on day 1. The intervention was focused on teaching meditation, the practice of mindfulness meditation following the procedure of each session lasted approximately $30 \mathrm{~min}$ (Chen et al., 2013).

Participants were taught how to concentrate on breathing with their eyes closed and to non-judgmentally become aware of their thoughts, feelings, and sensations while focusing on the flow of breath through the nostrils (Wallace, 2006). The underlying philosophy of mindfulness was also taught, emphasizing that participants should try to quiet the mind while paying attention to the breath and focusing on the dynamic sensations of the whole body (Wallace, 2006).

\section{DISCUSSION}

Students need to be prepared to manage their emotions from moment to moment while making clinical decisions and performing patient care. Appropriate management of their fluctuating emotions will enable students to effectively communicate with, and be supportive of, and future for patients with family members, ultimately providing safe patient care (Dubert et al., 2016). Given that nursing students need to pay attention to how they feel emotionally and regulate their emotions while caring for their patients, identifying those factors that help to cultivate this ability is critically important. Unfortunately, nursing students are not routinely taught how to manage the emotional 
HOLISTIK NURSING AND HEALTH SIENCE 1, (1), 2018 23-33

demands and challenges they experience in the clinical environment (Dubert et al., 2016).

To best prepare nursing students, tailored mindfulness-based interventions may help them learn to manage their emotions better during clinical practice. Mindfulness may buffer against the emotional demands of school and enhance the nursing student's awareness of emotions and feelings (Bishop \& Bishop, 2004).

There are several core constructs of mindfulness. These constructs are control of attention, awareness, nonreactivity, and non-judgmental thoughts. Mindfulness training is thought to begin with attention or "paying attention on purpose" and is the foundation of mindfulness (Kabat-zinn, 2003). This is the deliberate act of focusing our minds on passing thoughts, feelings, emotions, and actions (Kabat-zinn, 2003). Kabat Zinn (2003) stated that mindfulness was not only "paying attention on purpose" but also paying attention to the present moment and paying attention non-judgmentally. Mindfulness training in attention is thought to increase both emotional and cognitive flexibility, allowing practitioners to develop a non-judgmental awareness (Bamber \& Schneider, 2016).

Mindfulness meditation is a method that may improve coping skills to enhance cognitive functioning, reduce stress, and improve mood in nursing students. Learning mindfulness meditation strategies can increase one's mindfulness in everyday life to improve health (Spadaro \& Hunker, 2016). As reported in the Spadaro \& Hunker (2016) study, stress was significantly reduced $(\mathrm{F}(2,24)=4.163, \mathrm{p}=.019)$. A decreasing trend for anxiety was noted with a significant difference between time points $(F(1,23)=$ $6.889, \mathrm{p}=.015)$ when mindfulness practice frequency was weekly to daily, cognition: ability to shift attention, attention selection, concentration, and accuracy improved.

If implemented and practiced in daily life by a student nurse, mindfulness will lead to higher self-concept. A higher self-concept was found to be directly related to progress academic learning. This report is supported by research from Khalaila (2014), who studied 170 nursing students and found that test result of anxiety and intrinsic motivation were found to be significant mediators of the relationship between selfconcept and academic achievement.

Most researchers reported significant decreases in stress and anxiety suggesting that MBSR and MM were successful in reducing college students' perceived stress and anxiety. And in the eight studies reviewed earlier, MBSR was reported as effective in decreasing stress and anxiety in the studies reviewed. MM was reported as effective in decreasing stress (self-reported) and anxiety the studies reviewed.

The main challenge for educators is to assist students in becoming successful in college. Nursing programs and university officials continue to be concerned with the ability of nursing students to successfully complete rigorous and challenging programs (Floyd et al., 2015). Based on the researched information presented about how mindfulness has benefitted both institution school of nursing and the nursing student population for the reduction in anxiety, it can be concluded that this type of holistic therapeutic 
intervention among nursing students could be a useful tool that supports mindfulness as a positive precursor to support academic learning and overall student performance (Manocchi, 2017).

\section{CONCLUSION}

During this review, it became clear to us that the nursing students should consider how to best prepare for the emotional and cognitive demands of school and their clinical experiences as a professional nurse after graduation (Dubert et al., 2016). So we conclude from this review that mindfulness can be an instrument to overcome these problems.

Mindfulness is a non-pharmacological approach and is part of holistic nursing. Mindfulness can be practiced virtually at anytime and anywhere and can impact significantly to improve mental health and decrease anxiety in nursing students. It can support student's learning productivity to improve academic achievement. We suggest that the use of mindfulness programs could be expanded to medical students or other health providers including the practicing nurses, doctors and so on, and to improve the psychosocial status and mindful attentiveness to patients' needs.

For future study, we hope that this research continues to reveal the influence of mindfulness on a spiritual approach. It is expected that by combining mindfulness therapy with a spiritual approach would be a good framework to nursing students in an academic. Future research should further explore the long-term impact of mindfulness with the spiritual approach of the Islamic religion that impacts on student learning productivity and academic achievement.

\section{ACKNOWLEDGMENTS}

The researchers would like to thank the faculty members (Dr. Meidiana Dwidiyanti, S.Kp., MSc,; Ns. Sri Padmasari, S.Kp, MNS) who have provided their guidance in the completion of this study, and also the mindfulness team for their contributions to this review.

\section{REFERENCES}

ACHA-NCHA. (2013). Reference Group Executive Summary, Fall 2012. Retrieved from http://www.acha-ncha.org/docs/ACHA-NCHA-II_ReferenceGroup_

Alzayyat, A., \& Al-Gamal. (2014). A review of the literature regarding stress among nursing students during their clinical education, (1976), 1-10.

Bamber, M. D., \& Schneider, J. K. (2016). Mindfulness-based meditation to decrease stress and anxiety in college students: A narrative synthesis of the research. Educational Research Review, 18, 1-32. https://doi.org/10.1016/j.edurev.2015.12.004

Beddoe, A. E., \& Murphy, S. O. (2004). Does mindfulness decrease stress and foster empathy among nursing students? The Journal of Nursing Education, 43(7), 305312. 
Bishop, S. R., \& Bishop, S. R. (2004). Mindfulness : A Proposed Operational Definition Mindfulness : $\quad$ A Proposed Operational Definition. https://doi.org/10.1093/clipsy/bph077

Chen, Y., Yang, X., Wang, L., \& Zhang, X. (2013). Nurse Education Today A randomized controlled trial of the effects of brief mindfulness meditation on anxiety symptoms and systolic blood pressure in Chinese nursing students. YNEDT, 33(10), 1166-1172. https://doi.org/10.1016/j.nedt.2012.11.014

Cook, L. J. (2005). Inviting teaching behaviors of clinical faculty and nursing students' anxiety. The Journal of Nursing Education, 44(4), 156-161.

Dawood, E. (2016). Relationship between Test Anxiety and Academic Achievement among Undergraduate Nursing Students. Journal of Education and Practice, 7(2), $57-65$.

Dubert, C. J., Schumacher, A. M., Jr, L. L., Gutierrez, A. P., \& Barnes, V. A. (2016). Mindfulness and Emotion Regulation among Nursing Students : Investigating the Mediation Effect of Working Memory Capacity. Mindfulness. https://doi.org/10.1007/s12671-016-0544-6

Floyd, J., Clarke, M., Ed, D., Clarke, M., \& Ed, D. (2015). We hereby recommend that the Dissertation by to Grade Point Average.

Kabat-zinn, J. (2003). Mindfulness-Based Interventions in Context : Past, Present , and Future, (2002), 144-156. https://doi.org/10.1093/clipsy/bpg016

Khalaila, R. (2014). Nurse Education Today The relationship between academic selfconcept, intrinsic motivation, test anxiety, and academic achievement among nursing students: Mediating and moderating effects. YNEDT, 1-7. https://doi.org/10.1016/j.nedt.2014.11.001

Li, G., Yuan, H., \& Zhang, W. (2015). Archives of Psychiatric Nursing The Effects of Mindfulness-Based Stress Reduction for Family Caregivers : Systematic Review. Archives of Psychiatric Nursing, https://doi.org/10.1016/j.apnu.2015.08.014

Manocchi, P. E. (2017). Fostering academic success in nursing students through mindfulness: A literature review. Teaching and Learning in Nursing. https://doi.org/10.1016/j.teln.2017.05.002

Melo, K., Williams, B., \& Ross, C. (2010). Nurse Education Today The impact of nursing curricula on clinical practice anxiety. YNEDT, 30(8), 773-778. https://doi.org/10.1016/j.nedt.2010.02.006

Platt, L. M., Whitburn, A. I., Platt-Koch, A. G., \& Koch, R. L. (2016). Nonpharmacological Alternatives to Benzodiazepine Drugs for the Treatment of Anxiety in Outpatient Populations: A Literature Review. Journal of Psychosocial Nursing and Mental Health Services, 54(8), 35-42. https://doi.org/10.3928/02793695-20160725-07

Pryjmachuk, S., \& Richards, D. A. (2007). Copyright (C) The British Psychological Society Predicting stress in pre-registration nursing students Copyright (C) The British Psychological Society, 125-144. https://doi.org/10.1348/135910706X98524

Sanko, J., Dnp, M. M., \& Ms, S. R. (2016). Nurse Education Today Exploring the impact of mindfulness meditation training in pre-licensure and post graduate nurses. YNEDT, 45, 142-147. https://doi.org/10.1016/j.nedt.2016.07.006 
Shapiro, A. L. (2014). Test anxiety among nursing students: A systematic review. Teaching and Learning in Nursing, 9(4), 193-202. https://doi.org/10.1016/j.teln.2014.06.001

Shapiro, S. L., Schwartz, G. E., \& Bonner, G. (2013). Effects of Mindfulness-Based Stress Reduction on Medical and Premedical Students - Shapiro Schwartz Bonner 1998.doc.pdf, 21(6), 581-599. Retrieved from http://www.openground.com.au/articles/Shapiro Schwartz Bonner 1998.doc.pdf

Sik, Y., Young, S., \& Ryu, E. (2009). Nurse Education Today The effectiveness of a stress coping program based on mindfulness meditation on the stress, anxiety, and depression experienced by nursing students in Korea. Nurse Education Today, 29(5), 538-543. https://doi.org/10.1016/j.nedt.2008.12.003

Song, Y., \& Lindquist, R. (2015). Effects of mindfulness-based stress reduction on depression, anxiety, stress and mindfulness in Korean nursing students. Nurse Education Today, 35(1), 86-90. https://doi.org/10.1016/j.nedt.2014.06.010

Spadaro, K. C., \& Hunker, D. F. (2016). Exploring The effects Of An online asynchronous mindfulness meditation intervention with nursing students On Stress, mood, And Cognition: A descriptive study. Nurse Education Today, 39, 163-169. https://doi.org/10.1016/j.nedt.2016.02.006

Sun, F. K., Long, A., Tseng, Y. S., Huang, H. M., You, J. H., \& Chiang, C. Y. (2016). Undergraduate student nurses' lived experiences of anxiety during their first clinical practicum: A phenomenological study. Nurse Education Today, 37, 2126. https://doi.org/10.1016/j.nedt.2015.11.001

Travis, \& Pearson. (2000). Pure Consciousness: Distinct Phenomenological and Physiological Correlates of "Consciousness Itself." The International Journal of Neuroscience, 100(1-4), 77-89.

Turner, K., \& Mccarthy, V. L. (2016). Stress and anxiety among nursing students: A review of intervention strategies in literature between 2009 and 2015. Nurse Education in Practice. https://doi.org/10.1016/j.nepr.2016.11.002

Wallace, B. A. (2006). The attention revolution: Unlocking the power of the focused mind. Wisdom $\quad$ Publications, 338 Retrieved from http://books.google.com/books?id=eSAUUapdbbIC\&pgis=1

Zeidan, F., Johnson, S. K., Diamond, B. J., David, Z., \& Goolkasian, P. (2010). Mindfulness meditation improves cognition : Evidence of brief mental training q. Consciousness and Cognition, 19(2), 597-605. https://doi.org/10.1016/j.concog.2010.03.014 